\title{
Assessment of Executive Functions in Preschool Children With Sickle Cell Anemia
}

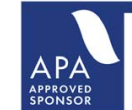

INS is approved by the American Psychological Association to sponsor Continuing Education for psychologists. INS maintains responsibility for this program and its content.

\author{
Michelle Downes, ${ }^{1,2}$ Fenella J. Kirkham, ${ }^{2}$ Paul T. Telfer, ${ }^{3}$ And Michelle de Haan ${ }^{2}$ \\ ${ }^{1}$ School of Psychology, University College Dublin, Dublin, Ireland \\ ${ }^{2}$ Developmental Neurosciences, UCL Great Ormond Street Institute of Child Health, London, United Kingdom \\ ${ }^{3}$ Department of Haematology, Barts Health NHS Trust, Royal London Hospital, London, United Kingdom \\ (Received August 8, 2017; Final Revision February 7, 2018; Accepted March 20, 2018; First Published Online May 30, 2018)
}

\begin{abstract}
Objectives: Children with sickle cell anemia (SCA) are commonly reported to experience executive dysfunction. However, the development of executive function (EF) in preschool-age children without stroke in this patient population has not been investigated so it is unclear when and how these deficits emerge. Methods: This case-control study examines the feasibility of assessing the early development of executive functioning in 22 preschool children years with SCA in the domains of processing speed, working memory, attention, inhibitory control, and cognitive flexibility, as well as everyday function, in comparison to matched control children. Results: A pattern of potential deficits in early emerging executive skills was observed in the domains of inhibitory control and cognitive flexibility. Parents reported no differences for everyday EF and no significant differences were observed for working memory and processing speed. Conclusions: Results suggest that deficits in everyday executive difficulties, working memory, and processing speed, as commonly reported for older children with SCA, may not yet have emerged at this early developmental stage, despite specific deficits in cognitive flexibility and inhibitory control on behavioral measures. The feasibility of using available executive measures with preschool age children to characterize the development of early EF skills is discussed. (JINS, 2018, 24, 949-954)
\end{abstract}

Keywords: Cognition, executive function, sickle cell disease, preschool, attention, cognitive flexibility

\section{INTRODUCTION}

Sickle cell anemia (SCA) is a genetic blood disorder that affects the ability of red blood cells to transport oxygen around the body. Stroke is common in SCA and typically occurs in the frontal cortex. However, for those patients who do not experience stroke, there remains evidence for bilateral cortical thinning and poorer white matter integrity which is thought to result from the effects of chronic hypoxia on the fronto-parietal regions that subsume executive functioning (Baldeweg et al., 2006).

Executive function (EF) is an umbrella term for a collection of skills that we use to coordinate and control our everyday behavior. The emergence of EF skills occurs alongside the protracted development of the prefrontal cortex with full maturity not reached until post-adolescence. Basic EF skills, such as attention control, are hypothesized to emerge first and lay down the foundation for more complex and later emerging

Correspondence and reprint requests to: Michelle Downes, School of Psychology, University College Dublin, Dublin 4, Ireland. E-mail: Michelle. Downes@ucd.ie
EF skills such as cognitive flexibility (Anderson, 2002). Improvements in attention control, switching, and fluency, are reported up to 6 years in typically developing children, with significant gains in planning and organization in midchildhood, and rapid development of information processing speed in the preadolescent stage (Anderson, 2002).

Children with early brain insult are sometimes described to "grow into" later emerging deficits when impairments are reported to emerge with brain development and as developmental expectations grow to incorporate higher-order skills such as planning, organizing, and problem-solving. The severity of EF dysfunction, the most common cognitive deficit in SCA, is related to increasing neurologic morbidity although poor EF remains in this patient population in the absence of neurologic morbidity (Berg, Edwards, \& King, 2012; Berkelhammer et al., 2007; Burkhardt et al., 2017; Hensler et al., 2014; Hijmans et al., 2011; Hollocks et al., 2012; Kral \& Brown, 2004; Nabors \& Freymuth, 2002; Smith \& Schatz, 2016).

It has been recommended that neuropsychological assessment of all children with SCA should include EF measures (Daly, Kral, \& Tarazi, 2011). However, until recently, a lack of age-appropriate measures for the preschool population has 
hindered research (Glass et al., 2012). Previous studies with preschool children with SCA have largely focused on global measures of cognition, showing evidence for early cognitive delay (Drazen, Abel, Gabir, Farmer, \& King, 2016; Glass et al., 2012; Tarazi, Grant, Ely, \& Barakat, 2007). The lack of focus on EF in preschool children makes it difficult to ascertain the extent of potential EF deficits at this early stage although there is emerging evidence for subtle delays in early working memory and processing speed (Hogan, Telfer, Kirkham, \& de Haan, 2013; Schatz \& Roberts, 2007).

Additional barriers to knowledge of EF development more generally in children and adults with SCA in previous studies include combined sickle cell genotypes and neurological histories, no matched controls, and insufficient descriptions of poor performance or measures (Burkhardt et al., 2017; Ruffieux et al., 2013; Smith \& Schatz, 2016). Clinicians, parents and educators tend to underestimate the rate of neurocognitive delay (Glass et al., 2012). Promoting EF at an early stage could reduce the achievement gap often reported for this patient population.

The aim of this study is to better establish the neuropsychological profile of EF in preschool-age children with SCA who have no clinical evidence of neurological morbidity while determining the feasibility of available EF measures for this age range. It is hypothesized that children with SCA will show EF deficits at this early stage that may not yet be observable in everyday contexts.

\section{METHODS}

\section{Participants}

Control children were recruited through the same clinics as patients at Barts NHS Trust ( $n=11$ siblings) or through study advertisement in local boroughs $(n=2)$. Patients were informed of the study by their consultant hematologist during their clinical visit if they met the following inclusionary criteria: aged between 36 and 72 months, HbSS genotype, no history of stroke/known neurological issues, normal transcranial Doppler (TCD), no developmental/psychiatric disorders, full-term delivery, and fluent in English. Inclusionary criteria for the matched control group were no history of developmental/psychiatric disorders, full-term delivery, fluent in English, Black British, and socioeconomic status (SES; by postcode to estimate total weekly house income).

The participation rate for those introduced to the study was approximately $85 \%$, there were no differences between those who chose not to participate. Reasons for non-participation included unavailability or a lack of response when contacted by the researcher. The 22 patients who participated in the study represented approximately $20 \%$ of the children within the same age range and with a diagnosis of SCA registered on the Barts NHS Trust database. Data on other inclusionary criteria for those on the database not participating in the study were not available. All patients had available TCD data with a mean delay between TCD and neuropsychological assessment of 60 days. No patients had abnormal transcranial Doppler recordings (velocity $>200 \mathrm{~cm} / \mathrm{s}$ ). Five patients were on transfusion and four were on hydroxyurea. No influence of treatment type was observed. Table 1 shows population descriptives.

\section{Procedure}

NHS ethical approval (13/LO/0962) and site-specific approval was obtained. Participant recruitment occurred from March 2014 to July 2015. Written consent was obtained from guardians. Child assent was obtained. The session took place at UCL Great Ormond Street Institute of Child Health. A revised Scrambled Boxes Working Memory Task was administered, followed by a revised Picture Deletion Task for Preschoolers (DDTP). The Wechsler Preschool and Primary Scales (WPPSI-III-UK) were administered before the EF Scale for Early Childhood and two National Institutes of Health Toolbox (NIHTB) tasks: Processing Speed and Inhibitory Control. Parents completed the Behavior Rating Inventory of EF-Preschool (BRIEF-P). All tasks, described in the supplementary section, were completed within $2.5 \mathrm{hr}$.

\section{Data Analysis}

Statistical analyses were conducted using SPSS version 21.0. Multivariate analysis of variance, independent $t$ tests, Fisher's exact tests, and $\chi^{2}$ analysis were used with a significance level of $p<.05$.

\section{RESULTS}

\section{Working Memory}

One patient found seven stimuli by the final trial (4.5\%), while five patients $(22.7 \%)$ found eight stimuli, and the rest

Table 1. Participant characteristics

\begin{tabular}{lccc}
\hline \hline & Patients & Controls & \\
& $M(S D)$ & $M(S D)$ & \\
Variable & $n=22$ & $n=13$ & $p$-Value \\
\hline Male, $n$ & 13 & 5 & .31 \\
Age & $4.8(0.9)$ & $4.9(0.9)$ & .35 \\
FSIQ & $98.6(11.4)$ & $100.9(10.4)$ & .55 \\
SES & & & .56 \\
$\quad$ Level 1 & 3 & 3 & \\
Level 2 & 3 & 1 & \\
Level 3 & 9 & 3 & \\
Level 4 & 3 & 3 & \\
Level 5 & 4 & 4 & \\
Black British, $\mathrm{n}$ & 22 & 13 & \\
\hline \hline
\end{tabular}

Office for National Statistic Web site data was used to analyse SES based on postcode to estimate total weekly house income on a scale from 1 (up to £520) to 5 (over £791).

$\mathrm{FSIQ}=$ Full-Scale Intellectual Quotient. 
found all nine. There was no group difference in success rates. Two control children $(15.4 \%)$ found eight stimuli whilst the rest found all nine stimuli $\left(\chi^{2}=.64 ; p=.72\right)$. Multivariate analysis of variance (ANOVA) found no statistically significant differences between groups for total trials or the number of consecutively correct trials $(F 2,32=.181$; $p=.84)$.

\section{Attention}

Four patients and one control child did not pass the practice phase of the DDTP and one control participant did not attempt the DDTP due to administrator error.

Three patients did not complete the task so scores for total time, omissions, and commissions, were pro-rated. For those who passed the practice phase, an ANOVA including the individual test phase factors (omissions, commissions, time to completion) found no overall group differences $(F 1,29=.338 ; p=.34)$. There were no group differences for motor speed. Commissions was higher for the patients ( $p=.04$; Table 2). More than 1.5 standard deviations (SDs) above the combined mean score for omissions/commissions in typically developing children $(M=33.1)$ was considered poorer than average performance in this study. Seven patients performed lower than average in comparison to three controls.

\section{Cognitive Flexibility}

An independent $t$ test found a main effect for group on the switching score, showing poorer cognitive flexibility in patients $(t(30)=2.5 ; p=.02$; Table 2$)$. Patients typically reached ceiling on level four of the task in comparison to the controls who typically reached level five (Table 2). Six patients $(30 \%)$ did not reach the normed average highest level for their age range in comparison to one control child $(8.33 \%)$. The task was not administered to two patients and one control due to time restrictions.

\section{Inhibitory Control and Processing Speed}

Patients had lower mean scores for inhibitory control $(t(30)$ $=.76 ; p=.38)$ and processing speed $(t(30)=.24 ; p=.27)$, but these did not reach significance (Table 2). However, when looking at individual scores, seven (35\%) patients were more than 1.5 SDs below the normative mean scores for processing speed in comparison to three $(23 \%)$ of the controls, while three $(15 \%)$ patients were more than $1.5 \mathrm{SD}$ below the normative mean for inhibitory control in comparison to none of the controls. Technical issues prevented two patients from completing the measure while one control participant did not compete these tasks due to time restrictions.

Table 2. Means and standard deviations on executive measures

\begin{tabular}{|c|c|c|c|c|c|c|}
\hline EF domain & Measure & Individual scores & $\begin{array}{l}\text { Patients } \\
M(S D)\end{array}$ & $\begin{array}{c}\text { Controls } \\
M(S D)\end{array}$ & $\begin{array}{l}p \text {-Value } \\
\text { (d) }\end{array}$ & $\begin{array}{l}\text { Statistical } \\
\text { test }\end{array}$ \\
\hline \multirow[t]{9}{*}{ Parent-report } & \multirow[t]{9}{*}{ BRIEF-P } & GEC & 53.95 (13.9) & $50.69(11.2)$ & $.29(0.3)$ & \multirow[t]{9}{*}{ MANOVA } \\
\hline & & ISCI & $54.19(13.2)$ & $49.30(11.3)$ & $.18(0.4)$ & \\
\hline & & EMI & $54.05(13.3)$ & $50.69(12.6)$ & $.37(0.3)$ & \\
\hline & & FI & $52.67(14.16)$ & $48.15(9.1)$ & $.18(0.4)$ & \\
\hline & & Inhibit & $52.19(11.8)$ & $50.30(11.1)$ & $.83(0.2)$ & \\
\hline & & Shift & $50.66(11.4)$ & $48.31(9.0)$ & $.11(0.2)$ & \\
\hline & & Emotional control & $54.38(14.17)$ & $48.30(10.4)$ & $.04(0.5)$ & \\
\hline & & Working memory & $54.43(12.3)$ & $51.61(11.5)$ & $.39(0.2)$ & \\
\hline & & Plan/organize & $52.86(13.9)$ & $49.31(13.1)$ & $.37(0.3)$ & \\
\hline \multirow{2}{*}{$\begin{array}{l}\text { Inhibitory control and } \\
\text { processing speed }\end{array}$} & \multirow[t]{2}{*}{ NIHTB } & Inhibitory control & $92.38(22.6)^{\mathrm{a}}$ & $98.45(21.8)^{\mathrm{a}}$ & $.38(0.3)$ & T-test \\
\hline & & Processing speed & $80.28(12.71)^{\mathrm{a}}$ & $85.9(14.9)^{\mathrm{a}}$ & $.27(0.4)$ & T-test \\
\hline EF domain & Measure & Individual Scores & $\begin{array}{c}\text { Patients } \\
\text { Mean (SD) }\end{array}$ & $\begin{array}{c}\text { Controls } \\
\text { Mean (SD) }\end{array}$ & $\begin{array}{l}\text { P-value } \\
\text { (d) }\end{array}$ & $\begin{array}{l}\text { Statistical } \\
\text { test }\end{array}$ \\
\hline \multirow[t]{4}{*}{ Attention } & \multirow[t]{4}{*}{ DDTP } & Commissions & 77.9 (111.2) & $13.6(22.7)^{b}$ & $.04(0.8)$ & \multirow[t]{4}{*}{ MANOVA } \\
\hline & & Omissions & $22.9(16.8)$ & $26.7(30.4)^{\mathrm{b}}$ & $.73(0.1)$ & \\
\hline & & Time to complete & $12.1(4.9)$ & $11.06(4.2)^{\mathrm{b}}$ & $.52(0.2)$ & \\
\hline & & Motor speed & $51.9(13.7)$ & $46.6(8.3)^{\mathrm{b}}$ & $.54(0.5)$ & \\
\hline Cognitive flexibility & EF Scale for Early Childhood & Switching score & $46.6(14.4)^{\mathrm{a}}$ & $56.9(9.7)^{\mathrm{a}}$ & $.02(0.8)$ & \\
\hline \multirow[t]{2}{*}{ Working memory } & \multirow[t]{2}{*}{ Scrambled Memory Task } & Total no. of trials & $14.68(3.92)$ & $14.64(3.99)$ & $.98(0.0)$ & \multirow{2}{*}{ MANOVA } \\
\hline & & Consecutively correct trials & $5.63(2.21)$ & $5.28(1.49)$ & $.67(0.2)$ & \\
\hline
\end{tabular}

${ }^{\mathrm{a}}$ Two patients and one control child did not complete the NIHTB tasks and the EF scale for Early Childhood.

${ }^{\mathrm{b}}$ One control child did not complete the DDTP.

$\mathrm{FI}=$ Flexibility Index. $\mathrm{d}=$ effect size; EMI = Emergent Metacognition Index; ISCI = Inhibitory Self-Control Index; MANOVA=multivariate analysis of variance. 


\section{Parent-Reports of Executive Functioning}

Multivariate analysis of variance on the five subdomains showed no overall group differences on the BRIEF-P $(F 1,35=.66 ; p=.66)$. There was a pattern of higher mean scores across all subdomains for the patients; however, this did not reach significance (Table 2). Clinically elevated General Executive Composite (GEC) scores were observed for three (14.3\%) patients and one (7.7\%) control.

\section{DISCUSSION}

The aim of this study was to investigate the EF profile of preschool children with SCA. Until recently, the lack of appropriate measures has been a barrier in the characterization of executive development in preschool children with SCA, despite widely reported executive deficits in older children (Berkelhammer et al., 2007). This study is the first robust assessment of individual EF domains in preschool-age children with SCA using available EF measures. Through the administration of standardized and lab-based measures, potential strengths and difficulties emerged for the preschool children with SCA. In particular, everyday EF (EF reported by parents as typically observed behavior in day-to-day real-life contexts), processing speed and working memory were relatively preserved whereas a pattern of specific deficits in cognitive flexibility and inhibitory control emerged on behavioral tasks.

Although more patients failed to retrieve all stimuli on the working memory task, the number of consecutively correct trials was comparable to controls. Contrary to current findings, previous studies have reported working memory deficits (Hijmans et al., 2011; Schatz \& Roberts, 2007). There were no significant differences observed between groups on NIHTB Processing Speed, or on the processing speed components of the DDTP (DDTP motor phase/ DDTP completion time) which is also in contrast to findings for school-age children (Burkhardt et al., 2017; Smith \& Shatz, 2016). In comparison to normative scores on NIHTB Processing Speed, both the patients and the controls showed poor processing speed, which may reflect the factors that they were matched on such as IQ or SES.

A pattern for difficulties with cognitive flexibility and inhibitory control was observed. The evidence for a specific deficit in cognitive flexibility builds upon previous research that observed poorer cognitive flexibility in older children (Hensler et al., 2014). In the current study, 30\% of patients did not reach the average performance level for their age range on the cognitive flexibility task (Fuglestad et al., 2014). Similarly, Hensler and colleagues (2014) reported that $45 \%$ of their school-age patients were impaired on a sorting task.

Potential deficits in inhibitory control were noted through the trend for poorer performance on NIHTB Inhibitory Control and the high error rate of DDTP commissions. The small group size that successfully completed the DDTP means that these findings must be interpreted cautiously and further research should address adapting the task further to capture low end performance. However, the higher level of commissions on this task adds weight to the trend for poorer performance on NIHTB Inhibitory Control.

The BRIEF-P GEC was more typical for the current patient population in comparison to previous reports for older children with SCA. Importantly, different subdomain categories on the school-age version of this measure preclude direct comparison with older patient groups. Hollocks and colleagues (2012) observed a higher mean composite score on the BRIEF $(M=62.2 ; S D=13.51)$ in 8- to 16-year-old children with SCA. Berg and colleagues (2012) also found a higher mean in 8- to 12-year-olds with SCA on both teacher $(M=59.1 ; S D=13.54)$ and parent $(M=52.5 ; S D=8.7)$ BRIEF reports. However, Hensler and colleagues (2014) found a more comparable mean to the current study in their cohort of 8- to 16-year-olds $(M=54.3 ; S D=14.4)$. Findings in the current study are also comparable to Kral and Brown (2004) as the group mean was also not in the clinical range in their study of 6- to 16-year-olds.

Everyday EF, as well as specific domains of working memory, attention, inhibitory control, cognitive flexibility, and processing speed, were measured in this study. Of interest, clinically elevated composite scores on the BRIEF-P captured patients who performed poorly on at least one behavioral task (Supplementary Table 1). This supports the utility of multi-method assessment of EF rather than a reliance on parent-report only to provide a holistic picture of an individual child. Findings on the BRIEF-P reflected a lower level of EF impairment in the patient group than what was observed on the other behavioral tasks. This could be attributed to subtle differences that are less readily observable in everyday contexts at this young age.

\section{Strengths and Limitations}

The generalizability of study findings is limited due to the small sample. The small control group limits statistical power. Effect sizes for each comparison have been included to assist with interpretation (Table 2). One strength of this study is the homogeneity of the patient population which overcomes the shortcomings of previous studies by focusing on the HbSS genotype and excluding patients with stroke, as well as a narrow age range. Group differences carry more weight as they are matched for age, ethnicity, gender, and SES, reducing the likelihood of spurious effects. This stringent approach allows us to elucidate differences due to disease without the influence of previously reported confounding factors.

Including control groups matched for ethnicity and SES is important as children with SCA are often from a minority group and face socioeconomic disadvantages known to influence EF (Yarboi et al., 2017). For example, the low group mean for the controls on NIHTB Processing Speed allowed us to better interpret findings that may have otherwise been construed as patients performing significantly poorer than peers. Order of administration may have impacted performance 
on tasks due to factors such as fatigue; however, this was controlled for with the comparison group.

Finally, incomplete batteries for some participants and the lack of large-scale normative data for some of the lab-based tasks, although widely used in developmental research, should also be considered in the interpretation of results. Further validation of the lab based EF measures, particularly the Scrambled Boxes task and the DDTP, are required in large populations and patient populations with well-known EF deficits. The failure of seven patients to pass the practice phase or complete the DDTP indicates that further task development is required to prevent floor effects in children with poorer attention control. Adapting the scoring system so that performance on the practice phase is incorporated could be one potential avenue to capture the range of performance at this level.

\section{CONCLUSION}

The findings of the current feasibility study inform researchers and clinicians on the potential impact of SCA in EF development and support early EF assessment. Recent research has highlighted a relation between poorer EF and increasing age and disease progression in older children and adults with SCA (Hijmans et al., 2011; Ruffieux et al., 2013; Vichinsky et al., 2010). Further research is necessary to develop valid EF measures for preschool children and to delineate the developmental trajectory of early EF in preschool children with SCA.

\section{ACKNOWLEDGMENTS}

The authors thank the families who participated in this study. This research was funded by the Child Health Research Charitable Incorporated Organisation and supported by the National Institute for Health Research Biomedical Research Centre at Great Ormond Street Hospital for Children NHS Foundation Trust and University College London. Dr. Kirkham was funded by the National Institute for Health Research for Patient Benefit Stream, Great Ormond Street Children's Charity, and Action Medical Research. None of the authors have potential conflicts of interest to be disclosed.

\section{SUPPLEMENTARY MATERIAL}

To view supplementary material for this article, please visit https://doi.org/10.1017/S1355617718000255

\section{REFERENCES}

Anderson, P. (2002). Assessment and development of executive functioning (EF) during childhood. Child Neuropsychology, 8(2), 71-82.

Baldeweg, T., Hogan, A.M., Saunders, D.E., Telfer, P., Gadian, D.G., Vargha-Khadem, F., \& Kirkham, F.J. (2006). Detecting white matter injury in sickle cell disease using voxel-based morphometry. Annals of Neurology, 59(4), 662-672.
Berg, C., Edwards, D.F., \& King, A. (2012). Executive function performance on the children's kitchen task assessment with children with sickle cell disease and matched controls. Child Neuropsychology, 18(5), 432-448.

Berkelhammer, L.D., Williamson, A.L., Sanford, S.D., Dirksen, C.L., Sharp, W.G., Margulies, A.S., \& Prengler, R.A. (2007). Neurocognitive sequelae of pediatric sickle cell disease: A review of the literature. Child Neuropsychology, 13(2), 120-131.

Burkhardt, L., Lobitz, S., Koustenis, E., Rueckriegel, S.M., \& Hernaiz, D.P. (2017). Cogntive and fine motor deficits in pediatric sickle cell disease cohort of mixed ethnic origin. Annals of Hematology, 96(2), 199-213.

Daly, D.B., Kral, M.C., \& Tarazi, R.A. (2011). The role of neuropsychological evaluation in pediatric sickle cell disease. The Clinical Neuropsychologist, 25(6), 903-925.

Drazen, C.H., Abel, R., Gabir, M., Farmer, G., \& King, A.A. (2016). Prevalence of developmental delay and contributing factors among children with sickle cell disease. Pediatric Blood \& Cancer, 63(3), 504-510.

Fuglestad, A.J., Whitley, M.L., Carlson, S.M., Boys, C.J., Eckerle, J.K., Fink, B.A., \& Wozniak, J.R. (2014). Executive functioning deficits in preschool children with fetal alcohol spectrum disorders. Child Neuropsychology, 21(6), 716-731.

Glass, P., Brennan, T., Wang, J., Luchtman-Jones, L., Hsu, L., Bass, C.M., ... Cheng, Y.I. (2012). Neurodevelopmental deficits among infants and toddlers with sickle cell disease. Journal of Developmental and Behavioral Pediatrics, 34(6), 399-405.

Hensler, M., Wolfe, K., Lebensburger, J., Nieman, J., Barnes, M., Nolan, W., ... Madan-Swain, A. (2014). Social skills and executive function among youth with sickle cell disease: A preliminary investigation. Journal of Pediatric Psychology, 39(5), 493-500.

Hijmans, C.T., Fijnvandraat, K., Grootenhuis, M.A., van Geloven, N., Heijboer, H., Peters, M., \& Oosterlaan, J. (2011). Neurocognitive deficits in children with sickle cell disease: A comprehensive profile. Pediatric Blood \& Cancer, 56(5), 783-788.

Hogan, A.M., Telfer, P.T., Kirkham, F.J., \& de Haan, M. (2013). Precursors of executive function in infants with sickle cell anemia. Journal of Child Neurology, 28(10), 1197-1202.

Hollocks, M.J., Kok, T.B., Kirkham, F.J., Gavlak, J., Inusa, B.P., DeBaun, M.R., \& de Haan, M. (2012). Nocturnal oxygen desaturation and disordered sleep as a potential factor in executive dysfunction in sickle cell anemia. Journal of the International Neuropsychological Society, 18(1), 168.

Kral, M.C., \& Brown, R.T. (2004). Transcranial Doppler ultrasonography and executive dysfunction in children with sickle cell disease. Journal of Pediatric Psychology, 29(3), 185-195.

Nabors, N.A., \& Freymuth, A.K. (2002). Attention deficits in children with sickle cell disease. Perceptual and Motor Skills, 95(1), 57-67.

Ruffieux, N., Njamnshi, A.K., Wonkam, A., Hauert, C.A., Chanal, J., Verdon, V., ... Ngamaleu, R.N. (2013). Association between biological markers of sickle cell disease and cognitive functioning amongst Cameroonian children. Child Neuropsychology, 19(2), 143-160.

Schatz, J., \& Roberts, C.W. (2007). Neurobehavioral impact of sickle cell disease in early childhood. Journal of the International Neuropsychological Society, 13(6), 933-943. 
Smith, K.E., \& Scahtz, J. (2016). Working memory in children with neurocognitive effects from sickle cell disease: Contributions of the central executive and processing speed. Developmental Neuropsychology, 41(4), 231-244.

Tarazi, R.A., Grant, M.L., Ely, E., \& Barakat, L.P. (2007). Neuropsychological functioning in preschool-age children with sickle cell disease: The role of illness-related and psychosocial factors. Child Neuropsychology, 13(2), 155-172.
Vichinsky, E.P., Neumayr, L.D., Gold, J.I., Weiner, M.W., Rule, R.R., Truran, D., ... McMahon, L. (2010). Neuropsychological dysfunction and neuroimaging abnormalities in neurologically intact adults with sickle cell anemia. JAMA, 303(18), 1823-1831.

Yarboi, J., Compas, B.E., Brody, G.H., White, D., Patterson, J.R., Ziara, K., \& King, A. (2017). Association of social-environmental factors with cognitive function in children with sickle cell disease. Child Neuropsychology, 23(3), 343-360. 